\title{
Twisting and Unknotting Operations
}

\author{
Yoshiyuki OHYAMA
}

\begin{abstract}
We define a twisting move, an $(n, k)$-move, on a link diagram and consider the question as to whether or not any two links are equivalent by this move. Moreover we show that any knot can be trivialized by at most twice twisting operations.
\end{abstract}

\section{INTRODUCTION}

In this paper, we define a twisting move on a link diagram, called an $(n, k)$-move, that is a $\pm k$-full twist operation of $n$ parallel strings. This move is related to unknotting operations closely. In other words an $(n, k)$-move induces some of unknotting operations. Then it arises a problem as to whether or not an $(n, k)$-move is an unknotting operation when natural numbers $n$ and $k$ are given. Moreover, if an $(n, k)$-move is an unknotting operation for some $n$ and $k$, can any $\mu$-component link be deformed into a trivial link by a finite sequence of $(n, k)$-moves?

As an answer of the above problem, we determine the number of equivalence classes of $\mu$-component links for an equivalence relation generated by an $(n, k)$-move except for some cases.

1991 Mathematjes Subject Classification: 57M25

Editorial Complutense. Madrid, 1994. 
In Section 4, we show that there exists a natural number $n$ such that any knot can be deformed into a trivial knot by an $(n, 1)$-move and an $(n-1,1)$-move. Namely, at most twice twisting operations can trivialize any knot. This is an answer for a problem given by Y. Mathieu [4].

\section{PRELIMINARY RESULTS AND $(n, k)$-MOVES}

R.H. Fox introduced the notion of congruence classes of knots in [3], and Y. Nakanishi and S. Suzuki showed the following result.

Definition 2.1 ([3]) Let $n$ and $q$ be non-negative integers. The knot, types $k$ and $\lambda$ are said to be congruent modulo $(n, q)$, written $k \equiv \lambda \bmod (n, q)$, if and only if there are knots $k_{0}, k_{1}, k_{2}, \ldots, k_{l}$ integers $c_{1}, c_{2}, \ldots, c_{l}$, and trivial knots $m_{1}, m_{2}, \ldots, m_{l}$ such that

(1) $k_{i \rightarrow 1}$ and $m_{i}$ are disjoint,

(2) $k_{i}$ is obtained from $k_{i-1}$ by $1 / c_{i} n$-surgery along $m_{i}$,

(3) the linking number $l k\left(k_{i-1}, m_{i}\right) \equiv 0 \bmod q$ and

(4) $k_{0}$ represents $k$ and $k_{\ell}$ represents $\lambda$.

Theorem 2.2 ([7]) Let $n$ be an integer greater than 1 and $q$ nonnegative integer such that $(n, q) \neq(2,1)$ nor $(2,2)$. For congruence modulo $(n, q)$, there exist infinitely many distinct classes.

Moreover, Nakanishi [8] [10] showed the following theorem in the case of $(n, q)=(2,1)$ and $(2,2)$.

Theorem $2.3([8],[10])$ All knot types are congruent modulo $(2,1)$ and modulo (2,2).

Fox's congruence classes are concerned with oriented knots. We can make the following definition for unoriented links.

Definition 2.4 Let $n$ and $k$ be non-negative integers. The link type $L^{\prime}$ is said to be obtained from $L$ by an $(n, k)$-move, if and only if there is a trivial knot $m$ which bounds a disk $D$, such that 
(1) $L$ and $m$ are disjoint,

(2) $L$ intersects $D$ transversely at $n$ points,

(3) $L^{\prime}$ is obtained from $L$ by $\pm 1 / k$-surgery along $m=\partial D$.

Obviously an $(n, k)$-move is the following local move on a link diagram and a $(1, k)$-move cannot change the link types.

Definition 2.4' For any integers $n(\geq 2)$ and $k(\geq 1)$, an $(n, k)$ move is a local move on a link diagnam depicted in Fig. 2.1.

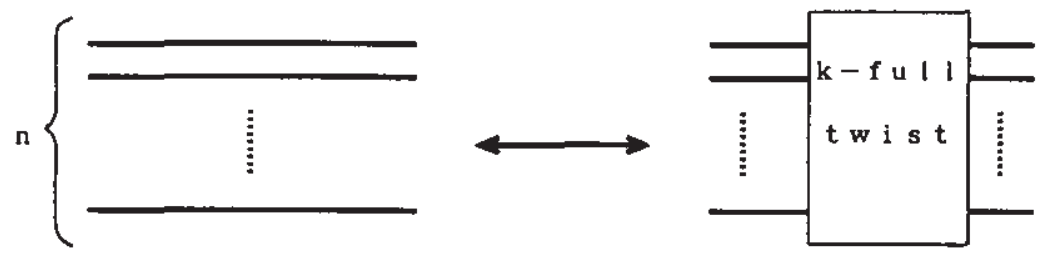

Fig. 2.1.

Remark 2.5 An $(n+2, k)$-move induces an $(n, k)$-move by joining two end points as is shown in Fig. 2.2.
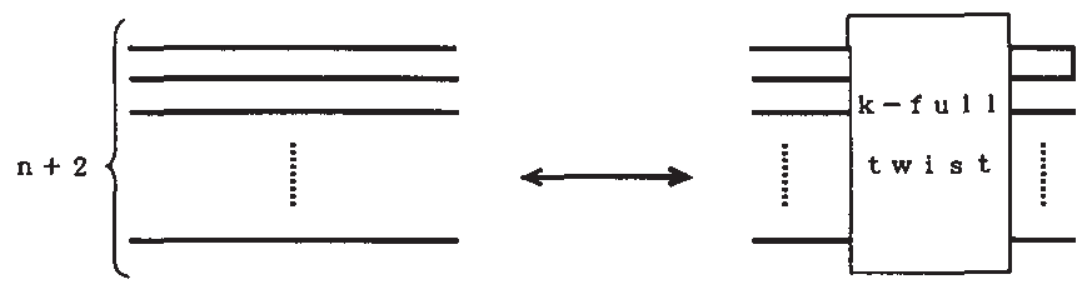

Fig. 2.2. 
We consider the problem as to whether or not an $(n, k)$-move is an unknotting operation. And if an $(n, k)$-move is an unknotting operation for some $n$ and $k$, can this move deform any $\mu$-component link into a trivial link? At first we make the following definition for local moves on a link diagram as $\mathrm{H}$. Aida did in [1] and [2].

Definition 2.6 (1) Two local move $A, B$ on a link diagram are locally equivalent, written $A \simeq \simeq^{\ell} B$, if and only if each move can be realized by a finite sequence of the other.

(2) Let $\mathcal{L}^{\mu}$ be the set of all $\mu$-component links. Two links $L_{1}, L_{2} \in \mathcal{L}^{\mu}$ are said to be $(n, k)$-equivalent if and only if $L_{1}$ is obtained from $L_{2}$ by a finite sequence of $(n, k)$-moves. $B y\left|\mathcal{L}^{\mu} /(n, k)\right|$, we denote the number of $(n, k)$-equivalence classes for $\mu$-component links.

Next we consider the relation between $(n, k)$-moves and some of unknotting operations.

We consider six replacements appearing in the Conway Third Identity. Let $L_{1}, L_{2}, L_{3}$ and $L_{4}$ be four links which differ only in one place as is shown in Fig. 2.3.

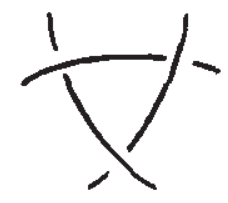

$\mathrm{L}_{1}$

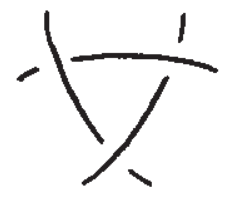

$\mathrm{L}_{3}$

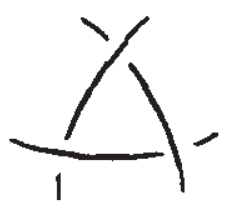

L 2

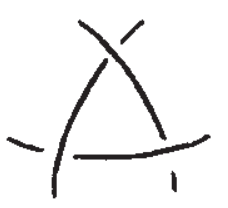

$\mathrm{L}_{4}$

Fig. 2.3. 
Nakanishi [9] defined a $\Delta_{i j}$-move as a local move between link diagrams of $L_{i}$ and $L_{j}$, and showed that each $\Delta_{i j}$-move is an unknotting operation. In our notation, Proposition 1 of [9] is stated as follow.

Proposition 2.7 ([9]) $\Delta_{i j}$-moves are classified to the following up to local equivalence.

(1) $\Delta_{12}$-move $\simeq^{\ell} \Delta_{34}$-move $\simeq^{\ell} \Delta_{13}$-move $\simeq^{\ell} \Delta_{24}$-move.

(2) $\Delta_{14}$-move $\simeq^{\ell} \Delta_{23}$-move.

Moreover Nakanishi proved the following proposition for $\mu$-component links.

Proposition 2.8 ([9]) For the equivalence relation generated by a $\Delta_{12}$-move, the number of equivalence classes for $\mu$-component links is $2^{\mu-1}$.

A $\Delta_{14}-\left(\Delta_{23}-\right)$ move is a $\Delta$-unknotting operation [6] and a $\Delta$ unknotting operation cannot change the linking number of a link.

A $\Delta_{i j}$-move is closely related to $(n, k)$-moves.

Proposition 2.9 (1) $A \Delta_{14}$-move is generated by a finite sequence of (2.2)- and (3,2)-moves.

(2) $\Delta_{12}$-move $\simeq^{\ell}(3,1)$-move.

\section{Proof.}

(1) is immediate from the proof of Theorem 2.3 in the case of modulo $(2,1)[8]$, and we have (2) from Fig. 2.4. 


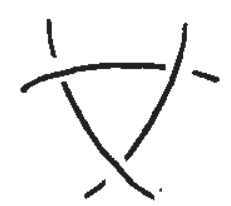

$\mathrm{L}_{1}$
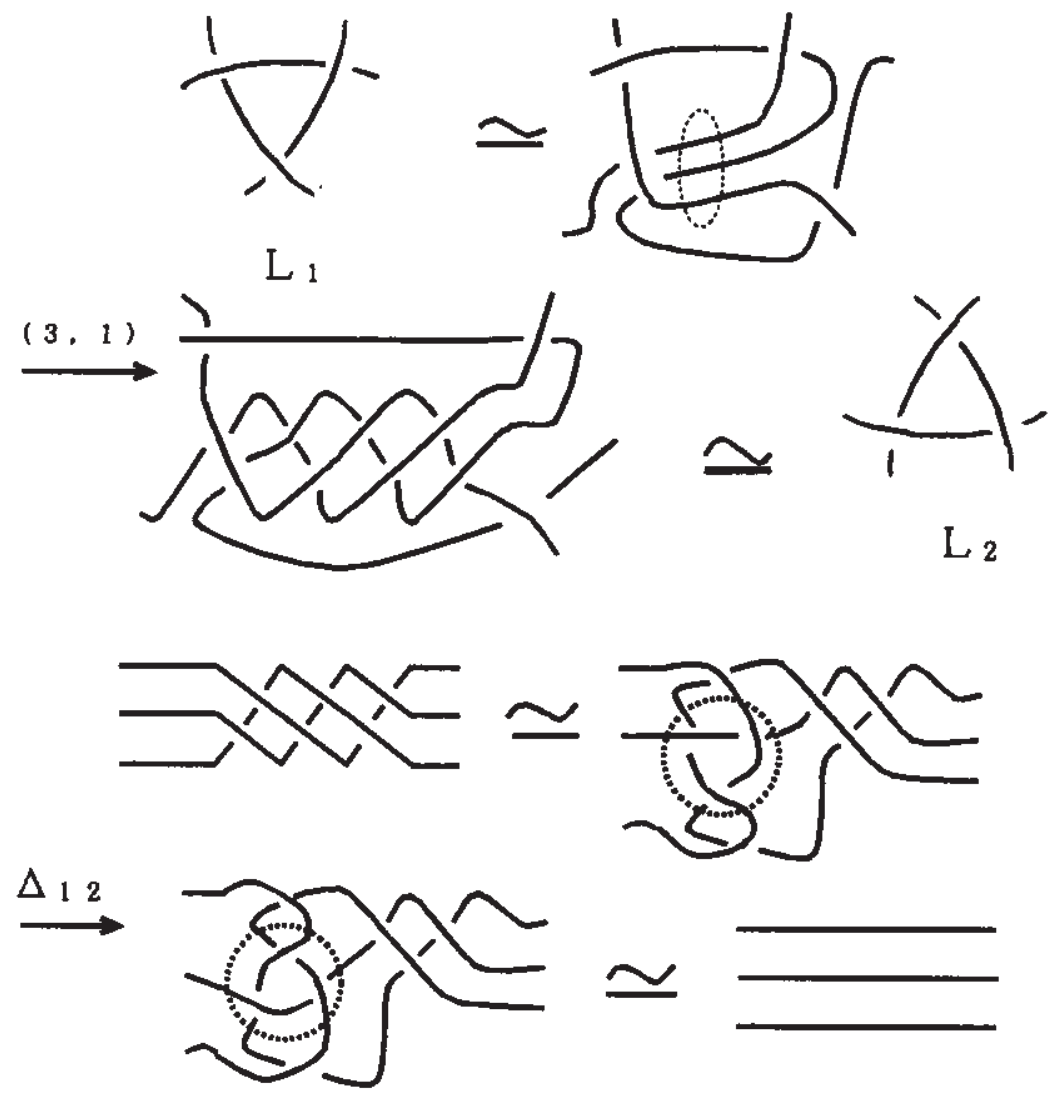

Fig. 2.4.

From Propositions 2.8 and 2.9, we have Corollary 2.10 .

Corollary 2.10 $\left|\mathcal{L}^{\mu} /(3,1)\right|=2^{\mu-1}$.

Murakami [5] defined a $\sharp$-unknotting operation which is a local move on a link diagram as illustrated in Fig. 2.5. 


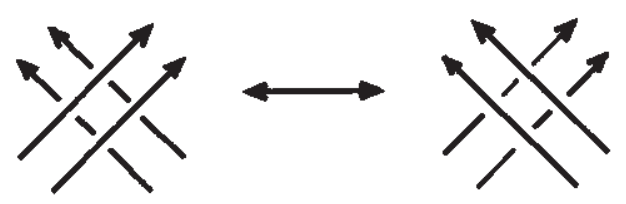

Fig. 2.5 .

By Fig. 2.6., we have the relation between a -unknotting operation and an $(n, k)$-move.

Proposition 2.11 Let $(3,1)$ '-move be an oriented $(3,1)$-move, where we give a parallel orientation. Then a -unknotting operation is locally equivalent to a $(3,1)$ '-move.
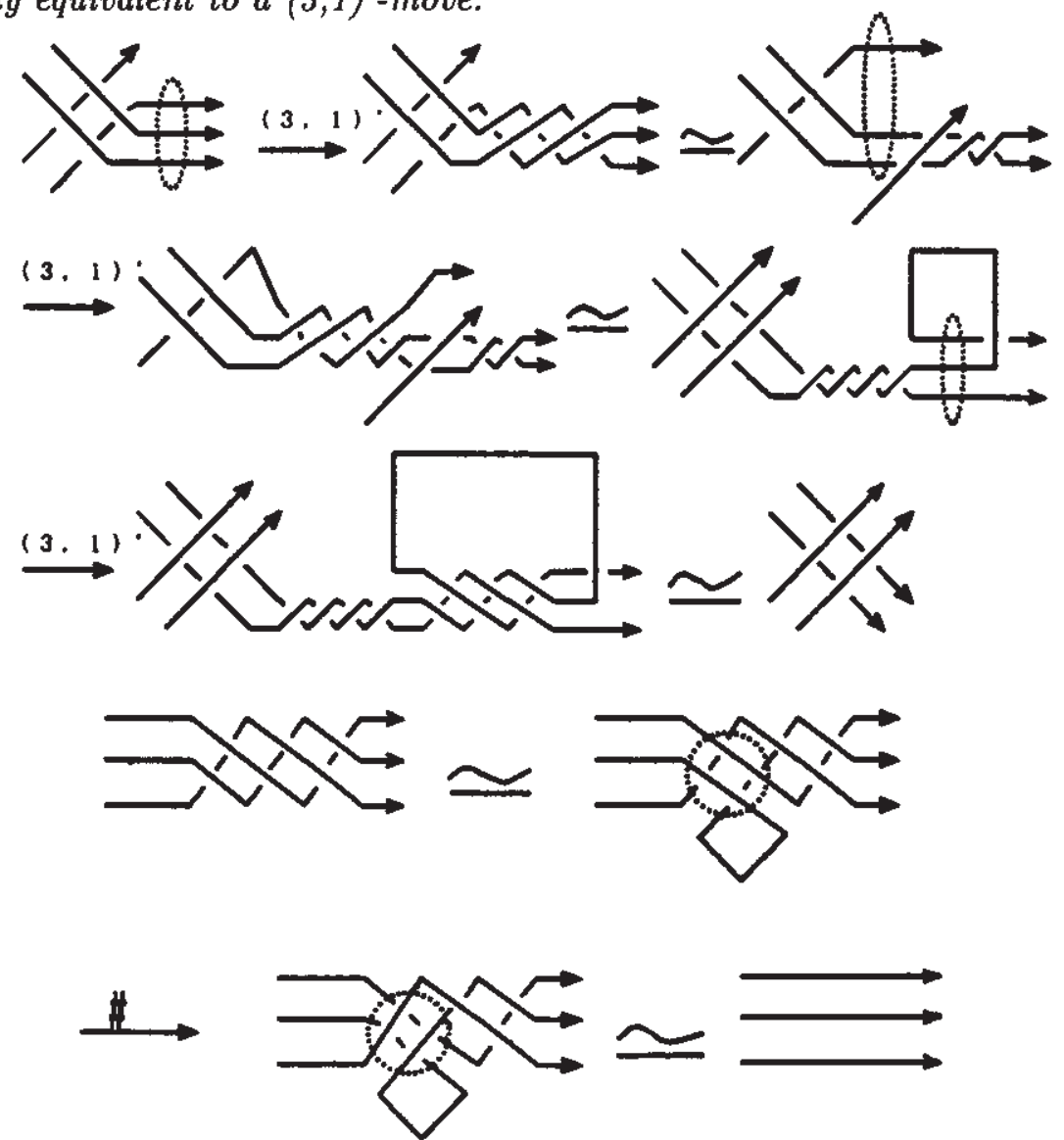

Fig. 2.6. 
From now, we consider an $(n, k)$-move from a view of local equivalence and determine the number of equivalence classes for knots and links.

Proposition 2.12 follows from Theorem 2.2.

Proposition 2.12. Let $\mathcal{K}$ be the set of all knots, then we have $|\mathcal{K} /(n, k)|=\infty$ for $k(\geq 3)$, which denotes that there exist infinitely many distinct classes.

By the above Proposition, if $k$ is greater than two, an $(n, k)$-move is not as unknotting operation, then it is enough to consider the cases $k=1$ and 2 .

Theorem 2.13 If $n$ is even, $(2,1)$-move $\simeq^{\ell}(n, 1)$-move, and if $n$ is odd, $(3,1)$-move $\simeq^{\ell}(n, 1)$-move.

Corollary 2.14 An (n,1)-move is an unknotting operation.

Proof of Theorem 2.13 is given in Section 3.

From Theorem 2.13 and Corollary 2.10, we can determine the number of equivalence classes for $\mathcal{L}^{\mu}$.

Corollary 2.15 If $n$ is even, $\left|\mathcal{L}^{\mu} /(n, 1)\right|=1$, and if $n$ is odd, $\left|\mathcal{L}^{\mu} /(n, 1)\right|=2^{\mu-1}$.

Next we consider the case $k=2$. If $n$ is even, the proof of Theorem 2.3 in [10] shows the following.

Proposition 2.16 If $n$ is even and is greater than or equal to 6 , an $(n, 2)$-move is an unknotting operation.

Suppose that $n$ is odd and that a knot $K^{\prime}$ is obtained from a knot $K$ by an $(n, 2)$-move. Let $G$ and $G^{\prime}$ be the Georitze matrices of $K$ and $K^{\prime}$, respectively. Wu showed that $G^{\prime} \cong G \oplus(-1)$ in the proof of Theorem 4 in [14]. Then if $n$ is odd, an $(n, 2)$-move cannot change the absolute value of the determinant of a knot. Therefore we have Theorem 2.17.

Theorem 2.17 If $n$ is odd and $\mathcal{K}$ is the set of all knots, then $|K /(n, 2)|=\infty$. 
Therefore, we determined the number of equivalence classes for $\mathcal{L}^{\mu}$ except for an $(n, 2)$-move where $n$ is even.

\section{PROOF OF THEOREM 2.13}

By Remark 2.5, if $n$ is even, an $(n, 1)$-move induces a $(2,1)$-move and if $n$ is odd, an $(n, 1)$-move induces a $(3,1)$-move obviously. Since a $(2,1)$-move is equivalent to a move changing a crossing, a $(2,1)$-move induces an ( $n, 1)$-move. Theorem 2.3 holds for an $(n, 1)$-move if $n$ is even. Therefore it is enough to show that a $(3,1)$-move induces an $(n, 1)$-move if $n$ is odd.

We will prove it by induction on $n$. Suppose a $(3,1)$-move induces a $(k, 1)$-move where $k$ is odd, then we show a $(3,1)$-move induces a $(k+2,1)$-move. A $(k+2,1)$-move is illustrated as in Fig. 3.1.

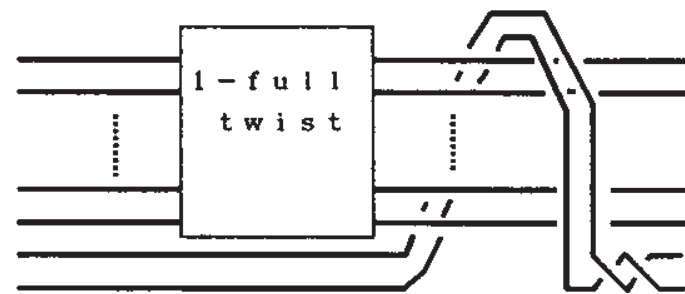

(a)

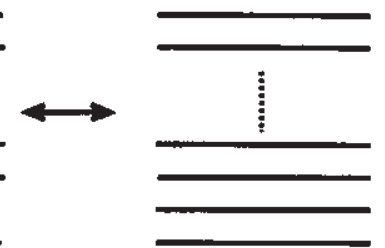

(b)

Fig. 3.1.

By the hypothesis of induction, Fig. 3.1(a) is deformed into Fig. 3.2 . by a finite sequence of a $(3,1)$-move. 


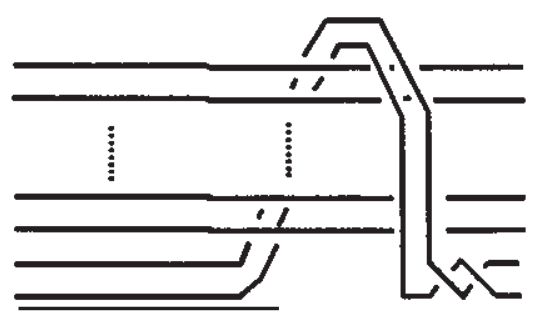

Fig. 3.2.

We call a move in Fig. 3.3. an unoriented $\Gamma$-move. $A(3,1)$-move induces an unoriented $\Gamma$-move as in shown in Fig. 3.4.

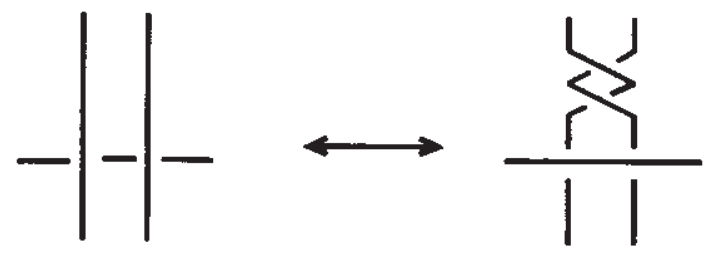

Fig. 3.3.

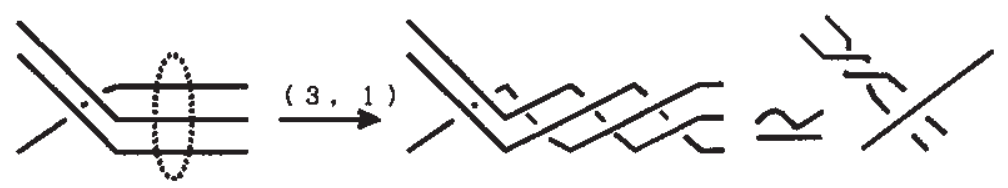

Fig. 3.4.

By performing an unoriented $\Gamma$-move on Fig. 3.2., we have Fig. 3.5. 


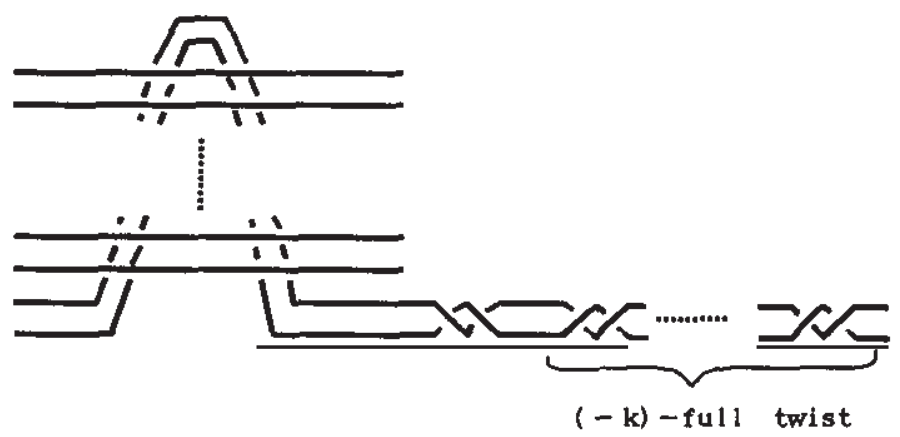

Fig. 3.5.

A $(3,1)$-move induces a $(2,2)$-move as shown in Fig. 3.6. Since $k$ is odd, Fig. 3.5. is deformed into a Fig. 3.1 (b) by $(2,2)$-moves. This completes the proof of Theorem 2.13 .
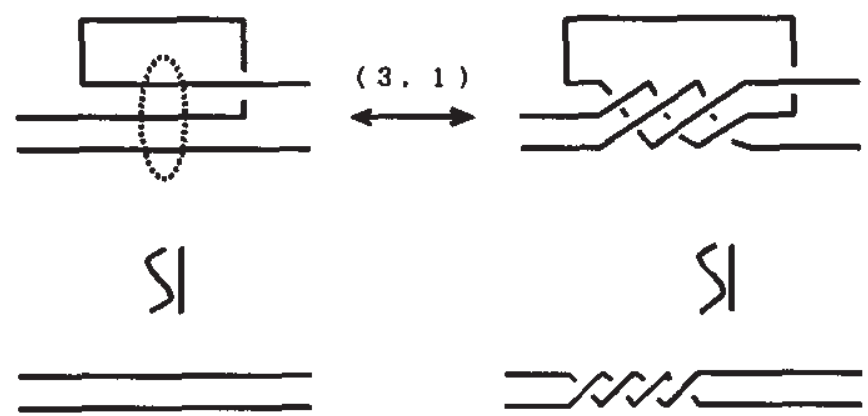

Fig. 3.6. 


\section{MATHIEU'S PROBLEM}

This section is concerned with the number of disks in Definition 2.4 which are necessary to deform any knot into a trivial knot. We call a disk $D$ in Definition 2.4 a surgery disk. Y. Mathieu [4] raised a following question.

Question If $K$ is any knot in $S^{3}$, can we find a surgery disk which trivializes $K$ ? If more than one disk is necessary to trivialize the knot, what about the minimal number of disks?

A. Yasuhara [13] and $\mathrm{K}$. Miyazaki give a negative answer for a first half of this question, independently. For a second half, we will show Theorem 4.1. Theorem 4.1 shows that if we choose two surgery disks suitably, we can trivialize any knot.

Theorem 4.1 For any knot $K$, there exists a naturăl number $n$ which satisfies the following sequence

$$
K \stackrel{(n, 1)}{\longrightarrow} K^{\prime(n-1,1)} \longrightarrow
$$

where $K^{\prime}$ is a knot and $O$ is a trivial knot, and by $K^{(n, 1)} K^{\prime}$, we denote that $K^{\prime}$ is obtained from $K$ by an $(n, 1)$-move.

To prove Theorem 4.1, we consider a certain canonical diagram of a knot which is given by S. Suzuki [11] at first and is arranged by M. Yamamoto [12].

Let $\sigma_{1}, \sigma_{2}, \sigma_{3}$ and $\sigma_{4}$ denote the upper, left, lower and right sides of a rectangle $\gamma_{0}$ respectively. Let $\gamma_{1}, \gamma_{2}, \ldots, \gamma_{u}$ denote trivial circles such that the diagram of $\gamma_{0} \cup \gamma_{1} \cup \ldots \cup \gamma_{u}$ is given as in Fig. 4.1. 


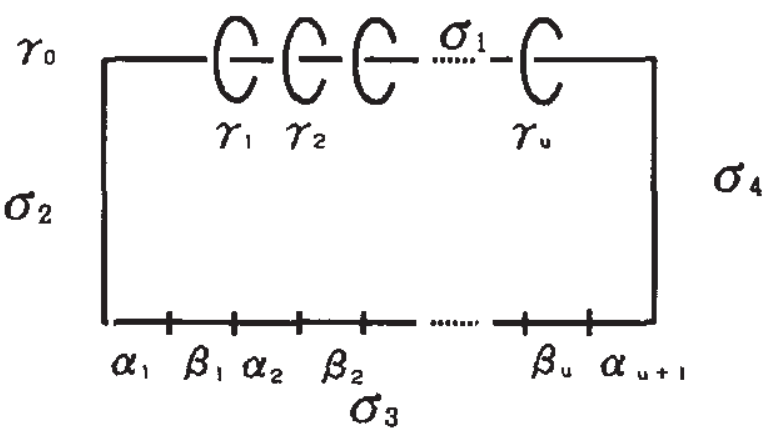

Fig. 4.1.

We divide $\sigma_{3}$ into $2 u+1$ subarcs $\alpha_{1}, \beta_{1}, \alpha_{2}, \beta_{2}, \ldots, \alpha_{u}, \beta_{u}$ and $\alpha_{u+1}$.

Lemma 4.2 ([12]) Let $\gamma_{0}, \gamma_{1}, \ldots, \gamma_{u}$ be as above. For any knot $K$ of the unknotting number at most $u$, there is a diagram of $K$ represented by $\gamma_{0}, \gamma_{1}, \ldots, \gamma_{u}$ and mutually disjoint strips $S_{1}, \ldots, S_{u}$ in $R^{3}$, satisfying the following conditions

(1) $\gamma_{0} \cap S_{i}=\gamma_{0} \cap \partial S_{i}=\beta_{i}$,

(2) $\gamma_{i}$ meets $S_{i}$ in an arc $\delta_{i}$ of $\partial S_{i}$,

(3) $\gamma_{i}$ does not meet $S_{j}$ if $i \neq j$,

(4) $\partial S_{i}$ does not cross $\gamma_{0}, \gamma_{1}, \ldots, \gamma_{u}$ other than $\alpha_{i}$ in the diagram, and (5) $K=\left(\gamma_{0} \cup \ldots \cup \gamma_{u} \cup \partial S_{1} \cup \ldots \cup \partial S_{u}\right)-\operatorname{Int}\left(\beta_{1} \cup \ldots \cup \beta_{u} \cup \delta_{1} \cup \ldots \cup \delta_{u}\right)$, where $i, j=1,2, \ldots, u$.

An example of a canonical diagram is illustrated in Fig. 4.2. 


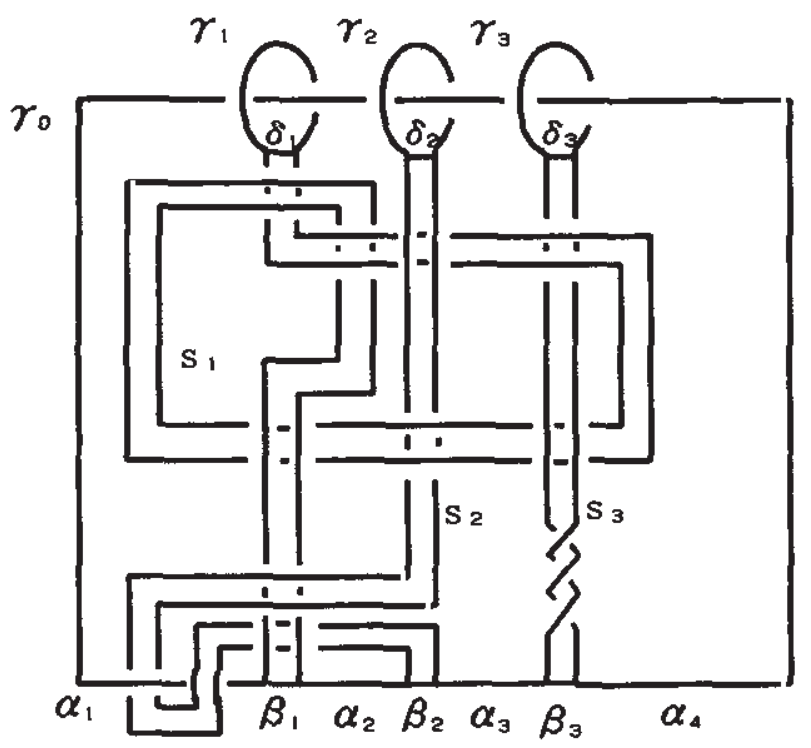

Fig. 4.2.

We pay attention to $\sigma_{1}$ and $\gamma_{i}(i=1,2, \ldots, u)$ and slide $\gamma_{i}$ into a position as is shown in Fig. 4.3(b).

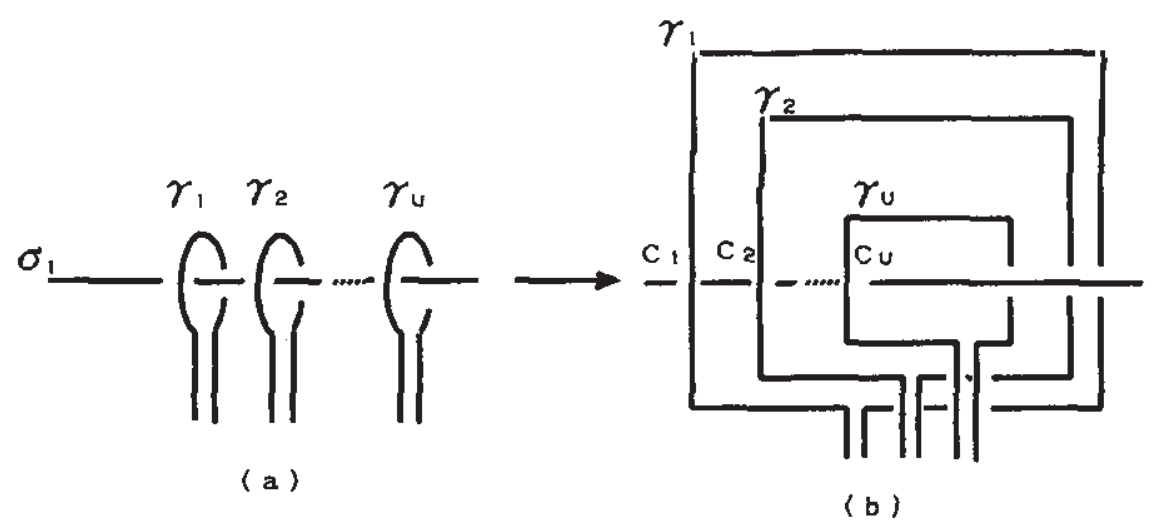

Fig. 4.3. 
And let $c_{i}$ be a crossing point such that $\gamma_{i}$ crosses $\gamma_{0}$ at $c_{i}$ as a overpass $(i=1,2, \ldots, u)$. If we change crossings at all $c_{i}$, then that diagram is deformed into a trivial knot diagram. Therefore we have Lemma 4.3.

Lemma 4.3 Let $K$ be a knot and $u$ the unknotting number of $K$. Then there exists a diagram $\bar{K}$ of $K$ such that by performing a local move in Fig. 4.4 on $\tilde{K}, \tilde{K}$ is deformed into a trivial knot diagram.

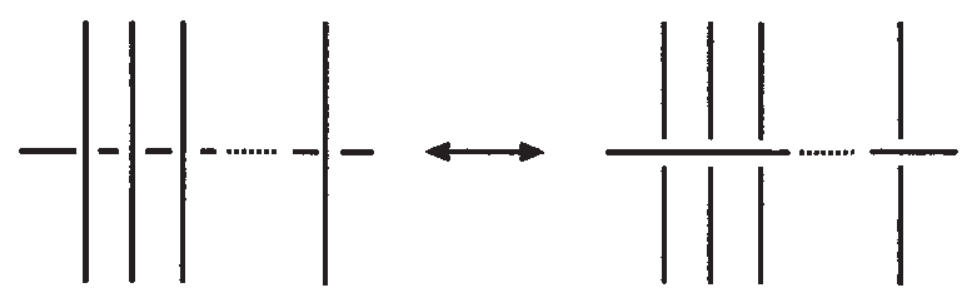

Fig. 4.4.

A local move in Fig. 4.4. is generated by a $(u+1,1)$-move and a $(u, 1)$ move as is shown in Fig. 4.5. Therefore we have the proof of Theorem 4.1 . 

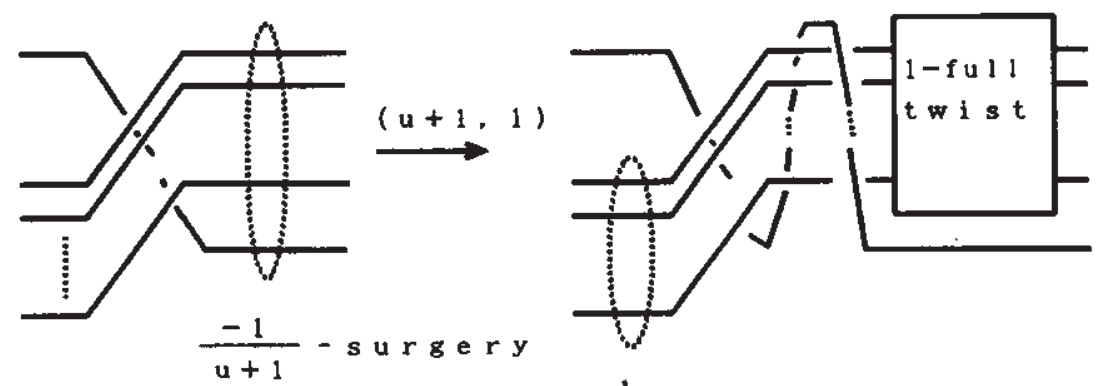

$\frac{1}{u}-s u r g e r y$

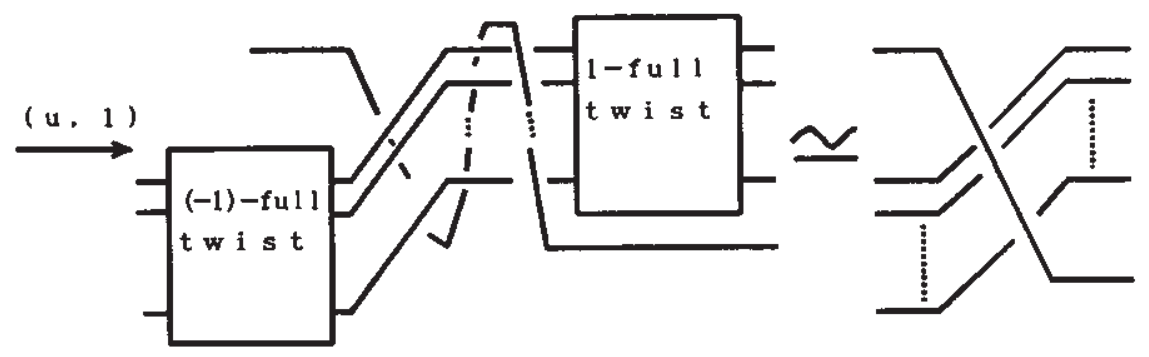

Fig. 4.5.

Acknowledgements. The author would like to express his appreciation to Prof. Katura Miyazaki for his valuable suggestions.

\section{References}

[1] Aida, H.: Unknotting operations of polygonal type. Tokyo J. Math., vol 15, No 1, (1992) 111-121.

[2] Aida, H.: The oriented $\Delta_{i j}$-move on links. Kobe J. Math., 9 (1992) 163-170. 
[3] Fox, R.H.: Congruence classes of knots. Osaka Math J., 10 (1958) $37-41$.

[4] Mathieu, $Y_{*}$ : Unknotting, knotting by twists on disks and property $(P)$ for knots in $S^{3}$. KNOTS90 (A. Kawauchi ed.), de Gruyter (1992) 93-102.

[5] Murakami, H.: Some metrics on classical knots. Math. Ann., 270 (1985) 35-45.

[6] Murakami, H. and Nakanishi, Y.: On a certain move generating linkhomology. Math. Ann., 284 (1989) 75-89.

[7] Nakanishi, Y. and Suzuki, S.: On Fox's congruence classes of knots. Osaka J. Math., 24 (1987) 217-225.

[8] Nakanishi, Y.: On Fox's congruence classes of knots II. Osaka J. Math., 27 (1990) 207-215.

[9] Nakanishi, Y.: Replacements of the Conway third identity. Tokyo J. Math, Vol 14 No. 1 (1991) 193-203.

[10] Nakanishi, Y.: From a view of localized link theory. KNOTS90 (A. Kawauchi ed.), de Gruyter (1992) 173-184.

[11] Suzuki, S.: Local knots of 2-spheres in 4-manifold. Proc. Japan Acad., 45 (1969) 34-38.

[12] Yamamoto, M.: Knots in spatial embeddings of the complete graph on four vertices. Topology and Its Applications. 36 (1990) 291-298.

[13] Yasuhara, A.: On slice knots in the complex projective plane. Rev. Mat. Complut. Madrid, vol 5 No. 2 (1992) 255-276.

[14] Wu, Y.: Signature of torus links. Kobe J. Math., 5 (1988) 297-304. 